\title{
Excision of Bone
}

National Cancer Institute

\section{Source}

National Cancer Institute. Excision of Bone. NCI Thesaurus. Code C51917.

The surgical removal of all or part of a bone. 